\title{
Intussusception of small intestine due to metastasis of large cell carcinoma of the lung with a rhabdoid phenotype
}

\author{
H. Otera*, F. Ikeda\#, S. Nakagawa\# , Y. Kono*, T. Sakurai*, K. Tada*, \\ K. Hashimoto" and A. Ikeda*
}

ABSTRACT: Large cell carcinoma of the lung with a rhabdoid phenotype is a rare type of lung cancer, and does not commonly metastasize to the small intestine. Herein we describe a 63-yr-old Japanese male with ileus resulting from small intestinal metastasis from lung cancer. Tumour enlargement was rapid and could not be treated with chemotherapy.

KEYWORDS: Large cell carcinoma, lung cancer, rhabdoid phenotype, small intestinal intussusception

$\mathbf{P}$ ulmonary carcinoma is the most frequently diagnosed major cancer, although only a few cases of large cell carcinoma of the lung with rhabdoid phenotype (LCCRP) have been reported [1]. Metastases to the brain, bone, adrenal glands and liver are more common in patients with lung cancer, whereas small intestinal metastasis is rare [2]. To our knowledge, there are no previous case reports describing intussusception of the small intestine caused by small intestinal metastasis of LCCRP.

\section{CASE REPORT}

A 63-yr-old male was admitted to the emergency department with continuous abdominal pain. He reported a 2-week history of vomiting at various times after meals. The abdomen showed distension and bowel sounds were absent. The patient was a smoker.

A routine chest radiograph taken as part of the initial work up showed a mass in the right lung (fig. 1a). Computed tomography (CT) scans demonstrated a mass in the right lung (fig. $1 \mathrm{~b}$ and c), intussusception of the small intestine (fig. 1d) and lymph node enlargement in the abdomen. Besides these findings, a significant abnormality was not detected.

During emergency surgery, the patient underwent segmental resection of the region of the small bowel showing intussusception along with the flexure of the small bowel. Furthermore, there were many small lesions in the small bowel that had not been detected on CT examination, indicating that the primary lesion was situated elsewhere. These additional lesions were not resected. We performed percutaneous needle biopsy of the lung lesion for diagnosis. On pathological examination of the resected small bowel tissue (fig. 2a) and the lung biopsy specimen (fig. $2 b$ and c), the microscopic appearances of these specimens were identical. These lesions were characterised histologically by the presence of typical rhabdoid cells, which are large cells with abundant cytoplasm and eccentric nuclei with central macronucleoli. In addition, these cells show rounded eosinophilic cytoplasmic inclusions. Based on these clinical findings, we diagnosed the patient as having primary lung tumour with metastasis to the small bowel.

Following surgical treatment the symptoms transiently recovered. However, the lung tumour quickly enlarged and pleural effusion developed within 3 weeks. Furthermore, the abdominal mass also increased and became more palpable, while the abdominal lymph nodes increased in size from $38 \times 43 \mathrm{~mm}$ to $71 \times 71 \mathrm{~mm}$. Chemotherapy was not administered due to the patients poor general condition and performance status. Moreover, radiation therapy was not applied to the abdominal lesions due to gastrointestinal bleeding. The patient did not receive any further treatment except pain control. He died 27 days after emergency surgery.

\section{DISCUSSION}

Lung cancer is the leading case of cancer death worldwide in both males and females. Common
AFFILIATIONS

Depts of *Respiratory Medicine, " Surgery, and

'Pathology, Nishi-Kobe Medical Center, Kobe, Japan.

CORRESPONDENCE

H. Otera

Dept of Respiratory Medicine

Nishi-Kobe Medical Center

5-7-1

Kojidai

Nishi-ku

Kobe

651-2273

Japan

E-mail: h.ohtera@nmc-kobe.org

Received:

Feb 162010

Accepted after revision:

March 162010

PROVENANCE

Submitted article, peer reviewed. 

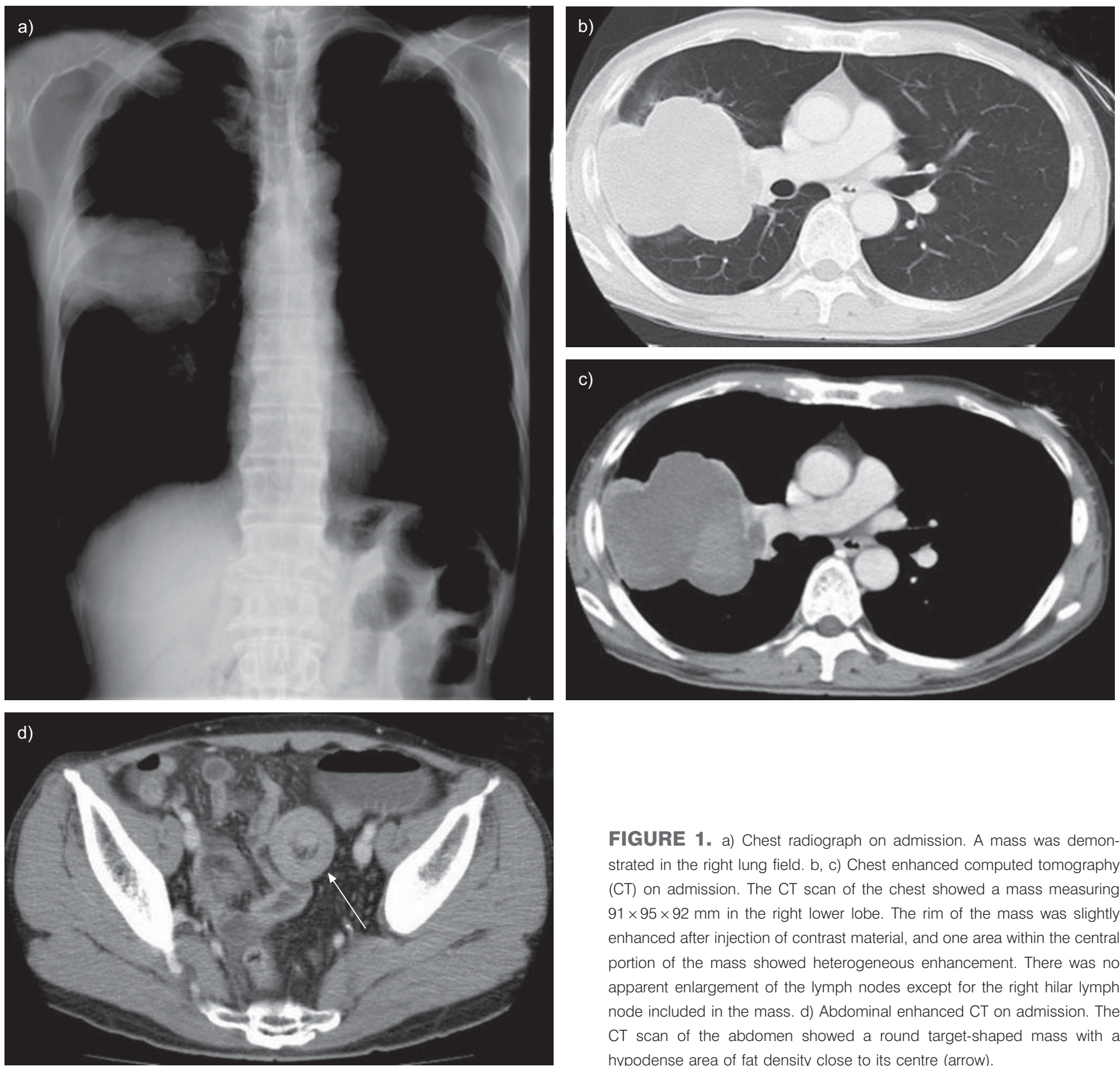

FIGURE 1. a) Chest radiograph on admission. A mass was demonstrated in the right lung field. b, c) Chest enhanced computed tomography (CT) on admission. The CT scan of the chest showed a mass measuring $91 \times 95 \times 92 \mathrm{~mm}$ in the right lower lobe. The rim of the mass was slightly enhanced after injection of contrast material, and one area within the central portion of the mass showed heterogeneous enhancement. There was no apparent enlargement of the lymph nodes except for the right hilar lymph node included in the mass. d) Abdominal enhanced CT on admission. The CT scan of the abdomen showed a round target-shaped mass with a hypodense area of fat density close to its centre (arrow).

sites of metastasis from lung cancer include the brain, bone, adrenal glands and liver. However, metastases to the small intestine are rare.

Primary rhabdoid tumour of the lung is a rare histological and clinical entity, and intussusception of the small intestine due to small intestinal metastasis from this cancer has not been reported previously. In the 1999 World Health Organization classification of lung tumours, the rhabdoid phenotype was included as a variant of large cell carcinoma [3]. BECKWITH and PALMER [4] first described malignant rhabdoid tumours in the kidneys of children in 1978. In 1995, the first definitive case of lung tumour showing rhabdoid morphology was described as a neuroendocrine carcinoma with a rhabdoid phenotype [5]. The rhabdoid cells of these tumours are characterised by the presence of a rounded eosinophilic cytoplasmic inclusion within large cells containing abundant cytoplasm and by the presence of a large eccentric nucleus with a central macronucleolus. Rhabdoid cells show an appearance similar to the cells of rhabdomyosarcoma [1].

Tumours with such a rhabdoid appearance have been described in many extra-renal sites. However, a lack of pathological uniformity in these cases has caused many researchers to doubt the concept of extrarenal rhabdoid tumours as a pathological diagnostic entity [6]. Metastases to the lung from rhabdoid tumours of the kidney and extrarenal rhabdoid tumours are a relatively common finding. Therefore, establishing a diagnosis of primary rhabdoid tumour of the lung requires extensive clinical, pathological and radiological 

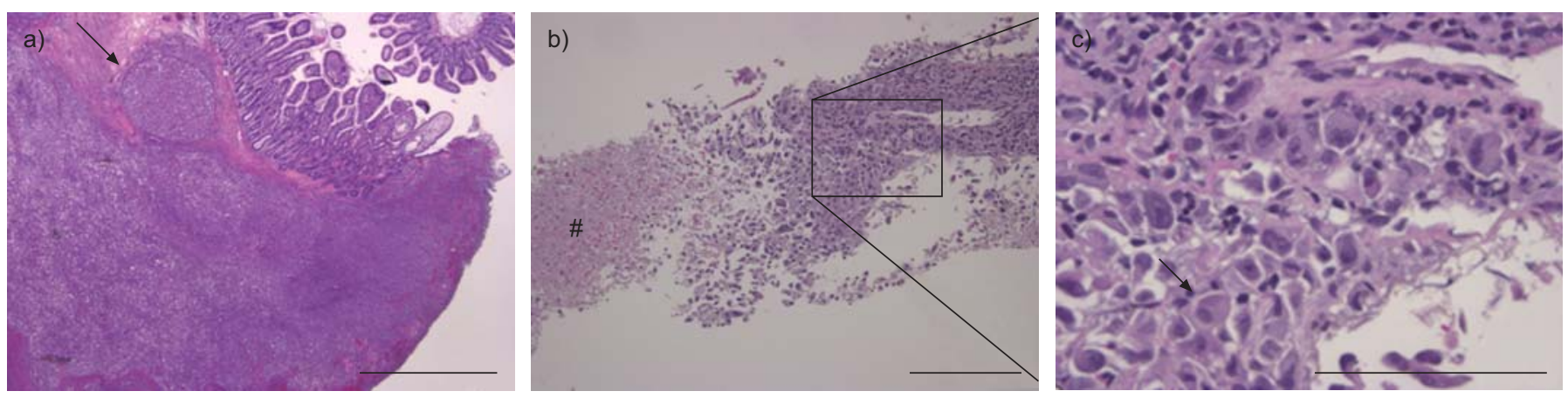

FIGURE 2. Histological findings. a) Small intestinal specimen obtained during surgery. Large tumour cells were mainly located in the submucosa and invaded the vessel (arrow) (haematoxylin and eosin stain.) b) Lung specimen obtained by percutaneous needle biopsy. Pathological findings of the specimen showed several large tumour cells similar to those in the intestinal lesion shown in (a). Abundant cytoplasm and eccentric nuclei and necrotic tissue (\#) were also noted (haematoxylin and eosin stain). c) Enlarged view of boxed section in (b) showing several large tumour cells (arrow). a) Scale bar $=2,000 \mu \mathrm{m}$, b) scale bar $=200 \mu \mathrm{m}, \mathrm{c}) \mathrm{scale}$ bar $=100 \mu \mathrm{m}$.

investigations in order to exclude a primary tumour located elsewhere [7]. Microscopically, most LCCRP are high grade or poorly differentiated, and show a combined mixed morphology with a rhabdoid component and an epithelial or mesenchymal component. These tumours usually express epithelial markers, such as cytokeratin and epithelial membrane antigen (EMA), and non-muscle mesenchymal cell markers such as vimentin. However, thyroid transcription factor-1 (TTF-1) is not expressed $[1,7,8]$, and is of limited value in determining the site of origin of an extrarenal rhabdoid tumour.

Histological findings of the resected small intestines in this case showed that the tumourous lesion was mainly located in the submucosa and invaded the vessels (fig. 2a). During surgery there were multiple tumour sites in the small intestine, suggesting metastases. Therefore, a primary tumour was suspected to be located elsewhere. The submucosal lesion at the flexure of the small intestine further supported this hypothesis. The larger metastatic lesion was considered to be the cause of intussusception. At present, positron emission tomography (PET) scanning is performed to determine malignancy, as well as staging of malignancy. SAINI et al. [1] reported the use of PET for LCCRP. However, we could not perform PET in this patient due to his poor condition. Based on the clinical findings, a diagnosis of primary lung tumour was accepted.

Intestinal metastasis from the lung is rare, whereas squamous cell carcinoma, poorly differentiated pulmonary adenocarcinomas and large cell carcinomas appear to be more common. However, digestive metastasis is more frequent than expected. As there are few symptoms of metastasis to the digestive tract, it is often difficult to diagnosis [2]. In our patient, the initial symptom was intussusception; therefore, we immediately selected surgical treatment.

$\mathrm{CT}$ is a useful pre-operative technique for adult intussusception [9]. Adult intussusception is considered a rare condition, accounting for $5 \%$ of all cases of intussusception and only 1$5 \%$ of intestinal obstructions. In contrast to those in children, cases of intussusception in adults are caused by secondary pathological conditions, such as tumours, metastatic neoplasms, etc. Therefore, surgical intervention is necessary [10]. We diagnosed intussusception in our patient based on CT findings. Surgical treatment was performed and his symptoms initially recovered. However, we found that the numerous other sites of small intestinal metastasis that had not been detected by preoperative CT. Surgery was useful for the treatment and detection of the extent of small intestinal metastases. LCCRP may be considered aggressive, as the majority of patients present with advanced disease. Thus, staging and identification of other metastatic lesions is important, and rapid diagnosis is better.

Including the present case, there are reports of 42 cases in the literature, which are summarised in table 1 . Lung tumours with rhabdoid morphology commonly occur in middle-aged to elderly adults presenting with haemoptysis and cough. Most of these cases presented at stage III or IV. Although patients with a short follow-up are included, this neoplasm is characterised by poor prognosis. Including our case, only six cases of digestive metastasis have been reported. Moreover, after expression of clinical symptoms of digestive metastasis, the prognosis is poor. In case 27, the patient died 5 months after gastrointestinal bleeding occurred. In case 39 , the patient died 2 months after abdominal pain and tarry stool developed. In case 40, the patient died 4 months after rectal bleeding appeared. More than $10 \%$ rhabdoid cells indicate a significantly poorer prognosis, while $<5 \%$ has a negligible effect on prognosis [8]. In the lung specimens from our patient, $>10 \%$ of cancer cells were identified as rhabdoid cells, indicating a very poor prognosis. The lung tumour and abdominal lymph nodes rapidly enlarged during the brief post-operative course. Only two reports (cases 21 and 41) showed satisfactory results following timely surgery and adjuvant chemotherapy with rigorous follow-up, but such cases are rare.

In our case, chest CT showed a lobulated well-defined mass, which differed from that usually observed in "standard" nonsmall cell lung cancer. There is no consensus regarding the typical findings of LCCRP. The pathological findings of these reports characterised the tumours, the tumours had a clear border but were not encapsulated [7, 17, 19, 20]. The cut surface of these specimens showed haemorrhage $[7,11,15]$ and necrosis $[7,11,14-16,18,19]$. Moreover, the tumour has a large area of central necrosis with a thin rim of variable tumour cells $[11,19]$. Further accumulation of cases is necessary.

In conclusion, lung tumours containing rhabdoid cells are uncommon. We should consider that a patient with LCCRP 


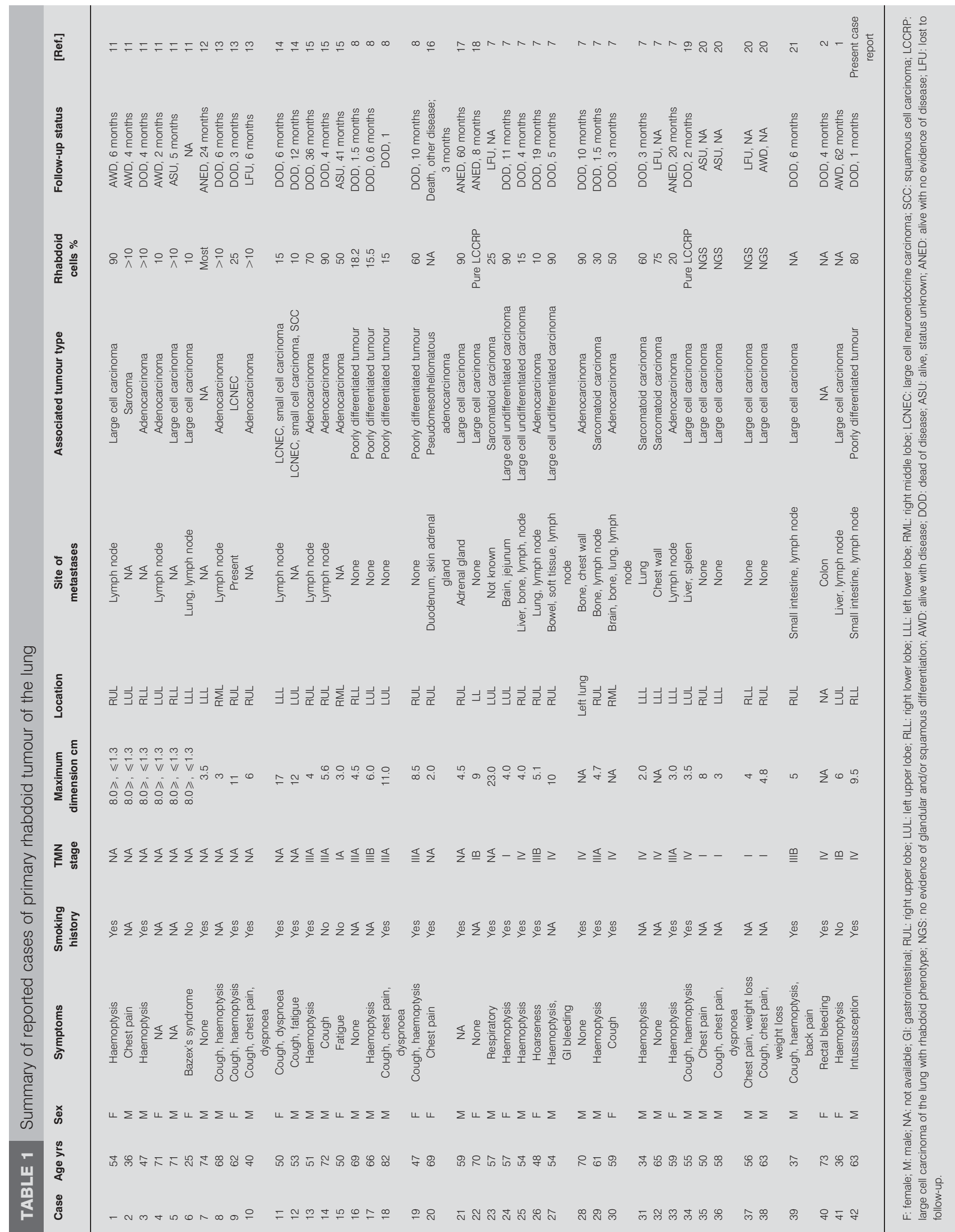


will show dynamic progression and has the possibility of metastasis to the digestive tract, as well as the more common metastatic sites. Early diagnosis facilitates intensive chemotherapy, and timely surgical treatment is needed.

\section{STATEMENT OF INTEREST}

None declared.

\section{REFERENCES}

1 Saini G, Kumar M, Julka PK, et al. Rhabdoid variant of lung cancer: clinicopathological details of a case and a review of literature. J Cancer Res Ther 2009; 5: 54-57.

2 Rossi G, Marchioni A, Romagnani E, et al. Primary lung cancer presenting with gastrointestinal tract involvement: clinicopathologic and immunohistochemical features in a series of 18 consecutive cases. J Thorac Oncol 2007; 2: 115-120.

3 Soft tissue tumours. In: Travis WD, Colby TV, Corrin B, et al., eds. World Health Organization International Histological Classification of Tumours. Histological Typing of Lung and Pleural Tumours. 3rd Edn. Berlin, Springer-Verlag, 1999; pp. 40-42.

4 Beckwith JB, Palmer NF. Histopathology and prognosis of Wilms tumors: results from the First National Wilms' Tumor Study. Cancer. 1978; 41: 1937-1948.

5 Colby TV, Koss MN, Travis WD. Carcinoid and other neuroendocrine tumors. In: Travis WD, ed. Tumors of the Lower Respiratory Tract. Washington, Armed Forces Institute of Pathology, 1995; p. 311.

6 Parham DM, Weeks DA, Beckwith JB. The clinicopathologic spectrum of putative extrarenal rhabdoid tumors: an analysis of 42 cases studied with immunohistochemistry or electron microscopy. Am J Surg Pathol 1994; 18: 1010-1029.

7 Tamboli P, Toprani TH, Amin MB, et al. Carcinoma of lung with rhabdoid features. Hum Pathol 2004; 35: 8-13.
8 Shimazaki H, Aida S, Sato M, et al. Lung carcinoma with rhabdoid cell: a clinicopathological study and survival analysis of 14 cases. Histopathology 2001; 38: 425-434.

9 Wang N, Cui XY, Liu Y, et al. Adult intussusception: a retrospective review of 41 cases. World J Gastroenterol 2009; 14, 15: 3303-3308.

10 Marinis A, Yiallourou A, Samanides L, et al. Intussusception of the bowel in adults: a review. World J Gastroenterol 2009; 28, 15: 407-411.

11 Cavazza A, Colby TV, Tsokos M, et al. Lung tumours with a rhabdoid phenotype. Am J Clin Pathol 1996; 105: 182-188.

12 Rubenchik I, Dardick I, Auger M. Cytopathology and ultrastructure of primary rhabdoid tumor of lung. Ultrastruct Pathol 1996; 20: 355-360.

13 Chetty R, Bhana B, Batitang S, et al. Lung carcinomas composed of rhabdoid cells. Eur J Surg Oncol 1997; 23: 432-434.

14 Chetty R. Combined large cell neuroendocrine, small cell and squamous carcinomas of the lung with rhabdoid cells. Pathology 2000; 32: 209-212.

15 Miyagi J, Tsuhako K, Kinjo T, et al. Rhabdoid tumor of the lung is a dedifferentiated phenotype of pulmonary adenocarcinoma. Histopathology 2000; 37: 37-44.

16 Attems JH, Lintner F. Pseudomesothelimatous adenocarcinoma of the lung with rhabdoid features. Pathol Res Pract 2001; 197: 841-846.

17 Kaneko T, Honda T, Fukushima M, et al. Large cell carcinoma of the lung with rhabdoid phenotype. Pathol Int 2002; 52: 643-647.

18 Hiroshima K, Shibuya K, Shimamura F, et al. Pulmonary large cell carcinoma with rhabdoid phenotype. Ultrastruct Pathol 2003; 27: 55-59.

19 Yilmazbayhan D, Ates LE, Dilege S, et al. Pulmonary large cell carcinoma with rhabdoid phenotype. Ann Diagn Pathol 2005; 9: 223-226.

20 Falconieri G, Moran CA, Pizzolitto S, et al. Intrathoracic rhabdoid carcinoma: a clinicopathological, immunohistochemical, and ultrastructural study of 6 cases. Ann Diagn Pathol 2005; 9: 279-283.

21 Goto $\mathrm{H}$, Ito $\mathrm{M}$, Yamaguchi $\mathrm{N}$, et al. [A case of large cell carcinoma of the lung with rhabdoid phenotype]. Nihon Kokyuki Gakkai Zasshi 2006; 44: 325-329. 